\title{
Indoor Experiments on Polarimetric SAR Interferometry
}

\author{
Lluís Sagués, Student Member, IEEE, Juan M. Lopez-Sanchez, Student Member, IEEE, \\ Joaquim Fortuny, Associate, IEEE, Xavier Fàbregas, Member, IEEE, Antoni Broquetas, Member, IEEE, and \\ Alois J. Sieber, Member, IEEE
}

\begin{abstract}
A coherence optimization method, which makes use of polarimetry to enhance the quality of SAR interferograms, has been experimentally tested under laboratory conditions in an anechoic chamber. By carefully selecting the polarization in both images, the resulting interferogram exhibits an improved coherence above the standard HH or VV channel. This higher coherence produces a lower phase variance, thus estimating the underlying topography more accurately. The potential improvement that this technique provides in the generation of digital elevation models (DEM) of non-vegetated natural surfaces has been observed for the first time on some artificial surfaces created with gravel. An experiment on a true outdoor DEM has not been accomplished yet, but the first laboratory results show that the height error for an almost planar surface can be drastically reduced within a wide range of baselines by using the optimization algorithm. This algorithm leads to three possible interferograms associated with statistically independent scattering mechanisms. The phase difference between those interferograms has been employed for extracting the height of vegetation samples. This retrieval technique has been tested on three different samples: maize, rice, and young fir trees. The inverted heights are compared with ground truth for different frequency bands. The estimates are quite variable with frequency, but their complete physical justification is still in progress. Finally, an alternative simplified scheme for the optimization is proposed. The new approach (called polarization subspace method) yields suboptimum results but is more intuitive and has been used for illustrating the working principle of the original optimization algorithm.
\end{abstract}

Index Terms-Digital elevation models, interferometry, inverse problems, radar polarimetry, synthetic aperture radar, vegetation.

\section{INTRODUCTION}

$\mathbf{S}$ ynthetic aperture radar (SAR) interferometry is an established technique based on combining two SAR images of the same scene acquired from slightly different viewpoints [1]. This technique is widely used for topographic mapping (DEM generation) and surface change detection (differential interferometry) [2], [3]. Moreover, in the past few years, SAR interferometry has also been used as an important tool to retrieve

Manuscript received May 3, 1999; revised November 30, 1999. This work was supported in part by the Spanish Commission for Science and Technology (CICYT) Grant TIC96-0879.

L. Sagués and X. Fábregas are with Departament de Teoria del Senyal i Comunicacions, Universitat Politècnica de Catalunya (UPC), Campus Nord UPC, 08034 Barcelona, Spain (e-mail: sagues@ voltor.upc.es).

J. M. Lopez-Sanchez, J. Fortuny, and A. J. Sieber are with the Space Applications Institute, Joint Research Centre of the European Commission, Ispra, Italy (e-mail: juanma-lopez@ieee.org; joaquim.fortuny@jrc.it).

A. Broquetas is with the Institut de Geomàtica, Parc de Montjuic, Barcelona, Spain (e-mail: toni@tsc.upc.es).

Publisher Item Identifier S 0196-2892(00)02085-4. physical parameters of terrestrial surfaces. Whatever the final application is, accurate interferograms are required. The quality of the interferograms is closely related to the correlation (coherence) between the two complex SAR images. If a high SNR is assumed and there are no changes in the scattering behavior of the measured scene between acquisitions (no temporal decorrelation), then the most important source of decorrelation is that produced by the separation between the two antennas (baseline decorrelation). The difference between the two look angles produces a shift and a stretch of the two imaged-terrain spectra. This spectral decorrelation can be compensated by shifting the transmitted central frequency during the second measurement or by removing the disjointed parts of both spectra [4]. However, the different heights of the scattering centers inside the resolution cell (volumetric effects) [5] remains as an important source of spectral decorrelation that cannot be compensated by filtering the two spectra, because they have different shapes. A possible way to reduce this decorrelation is to exploit multiple baseline takes [5], which implies collecting data from multiple surveys.

More recent studies have revealed that the spectral decorrelation can be reduced if fully polarimetric SAR data are collected. Radar polarimetry is a technique that has shown the ability to extract geophysical parameters from SAR images and its usefulness in terrain classification [6]-[8]. Polarimetry deals with the vector nature of the electromagnetic waves, and its strength resides in the physical meaning of the observables. The application of polarimetry to SAR interferometry has demonstrated the possibility of optimizing the coherence [9]. The idea of this optimization is to express the obtained SAR images in an arbitrary polarization basis, which is equivalent to selecting an arbitrary scattering mechanism in each image and to choose those scattering mechanisms that maximize the interferometric coherence.

The polarimetric optimization of the interferometric coherence has an exact formal solution, which was formulated in [9]. The optimization algorithm leads to two complex $3 \times 3$ eigenvalue problems that share the same eigenvalues. The three eigenvalues are related to pairs (one for each image) of eigenvectors, which can be interpreted as scattering mechanisms. All eigenvalues are real, and the optimum coherence is given by the highest one. Therefore, its corresponding interferometric phase can be used to generate the DEM of the scene with the highest coherence. Moreover, if the target morphology is equivalent to a multilayer structure (e.g. ground-trunks-branches-crown for forests), with each layer presenting a different scattering behavior, it has been postulated that the three optimum scattering 
mechanisms will be associated with the phase center of each layer. As a result, three interferograms could be computed, yielding the height of these layers. A possible application of this method is the estimation of vegetation height, since most plants can be modeled by a multilayer morphology. Note that some parts of the formal development of this method are explicitly repeated in this paper for completeness.

As will be described in this paper, an alternative way to implement this approach consists in looking at the possible coherence for every combination of elliptical polarizations and selecting the highest one. This simple procedure, called the polarization subspace method (PSM), is suggested to illustrate the procedure of choice of polarization states in a more intuitive way. However, the results obtained with this method are always suboptimum due to its intrinsic assumptions.

Since the early 1990's, a large amount of research on the estimation of vegetation height, biomass, and related parameters, by means of SAR data, has been carried out. A brief but well-oriented review on this topic can be consulted in [10]. Some recent approaches have made use of interferometry for estimating the canopy height by comparing the interferometric phase centers in the presence of vegetation with a previously known DEM of the area [11], [12]. Thus, the inverted height depends on the availability of a precise DEM of the area of interest. Another significant example of a combination of interferometric information (phase center and coherence) with backscattered power in order to invert characteristics of a layer of vegetation and the underlying topography was presented in [13]. The derivations of that paper are based on a simplified scattering model, enough to demonstrate the performance of that approach, but limited to represent all the fine characteristics of many vegetation volumes. An efficient way to circumvent these problems is the use of polarimetry. With the use of the polarimetric optimization, the estimation is performed by calculating the relative phase differences between polarization channels, and hence, no a priori information about the scene DEM is necessary. A scattering model is only necessary in the final conversion of the estimates into absolute heights.

To date, test sites presented in the literature with polarimetric SAR interferometry have been a mixed forestry/agricultural area close to Lake Baikal, measured at L band by SIR-C [9] and a test site with corn fields in Switzerland, measured at L band by E-SAR [14], [15]. In the first case, at the time of writing the first version of the present manuscript, no precise data about the scene were provided. However, a comparison with the actual ground truth was recently published in [16]. In the second case, an approximate height of about $2.2 \mathrm{~m}$ was estimated for corn fields, but no ground trutdata were available. Therefore, at the time of writing this paper, the first results of this technique to be compared with ground truth data are presented here.

This paper describes some laboratory experiments that have been carried out to show the remarkable contribution of polarimetry to SAR interferometry in the generation of accurate DEM's and retrieval of vegetation height. In order to have measurement conditions under control, the experimental validation was performed in an anechoic chamber. In conventional outdoor measurements, effects such as varying weather conditions, presence of RF interfering signals, unknown variations of the local topography, and soil conditions may heavily influence the data. As a result, experimental data acquired under these conditions may not be interpreted properly and consequently may lead to wrong conclusions. On the other hand, when running the measurements in an anechoic chamber under laboratory conditions, all relevant measurement parameters can be fully controlled and a complete and accurate analysis of an isolated sample can be carried out. We can also freely select the frequency range and incidence angles of interest. The radar backscatter can be accurately equalized by applying a single reference, fully polarimetric calibration [17], leading to a simpler interpretation of the results.

The objective of this paper is to demonstrate that under ideal conditions, the proposed algorithm yields the expected results. This laboratory test is an important step, previous to the application of the method in real conditions by using a spaceborne or airborne radar system. The relevance of the present work is not its direct applicability to outdoor configurations, but showing that this technique works properly in ideal conditions. Note that no thorough interpretation about the vegetation height estimates is given in this paper. This paper shows that the estimates are within the expected ranges (lower than the total heights) but the specific retrieved values are not justified. This justification is the subject of ongoing research.

The experiments were carried out in the anechoic chamber of the European Microwave Signature Laboratory (EMSL), JRC, Ispra, Italy [18]. The target used in the DEM reconstruction test was a gravel rough surface (roughness root mean square (RMS) of about $2 \mathrm{~cm}$ ) without slopes. Fully coherent, polarimetric data were collected at $\mathrm{X}$ band for different baselines. The retrieval of vegetation height was examined by measuring some vegetation samples at L, S, C, and X band. The targets were a maize sample, a rice sample, and a cluster of young fir trees.

The paper is organized as follows. In Section II, an expression of the interferometric coherence is provided for analyzing the degradation caused by different decorrelation sources, and two methods for improving the coherence are described: the wavenumber shift and the polarimetric optimization algorithm. Section III analyzes the experimental results obtained by applying these algorithms in laboratory conditions to DEM generation of nonvegetated natural surfaces. The use of the polarimetric optimization for extracting the height of vegetation samples is tested in Section IV. Finally, some conclusions are drawn in Section V.

\section{THEORY}

\section{A. Interferometric Coherence}

The geometry of a conventional interferometric imaging system is illustrated in Fig. 1. The interferogram is generated using two SAR images obtained from two slightly different viewing angles $\theta_{1}$ and $\theta_{2}$. The separation between the two antenna positions, $A_{1}$ and $A_{2}$, is known as the baseline distance $B$.

For the analysis, the problem is assumed to be invariant in the cross-range dimension, and we suppose that any resolution cell of the rough surface is formed by nondirectional, independent scatterers uniformly distributed along the $y$ and $z$ axes. The 


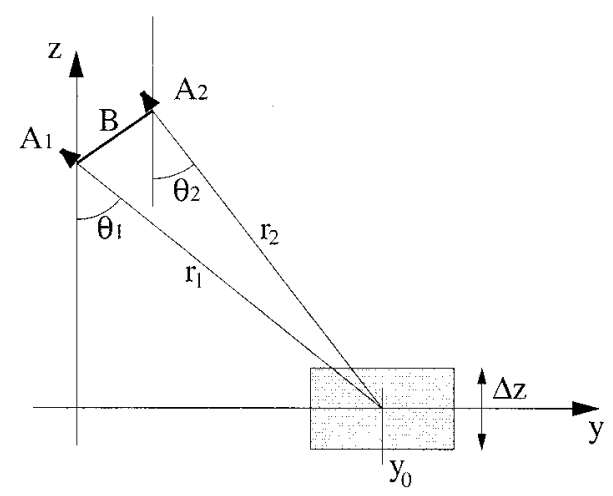

Fig. 1. Geometry of the interferometric SAR system.

following expression for the interferometric coherence between the two images from the same resolution cell has been derived [19]

$$
\gamma=\left(1-\frac{\Delta \theta}{\tan \theta} \cdot \frac{f_{0}}{W}\right) \operatorname{sinc}\left(2 \frac{f_{0}}{c} \cdot \frac{\Delta \theta}{\sin \theta} \Delta z\right)
$$

where $\theta$ is the mean look angle, $f_{0}$ is the central working frequency, $W$ is the frequency bandwidth, and $\Delta \theta=\theta_{1}-\theta_{2}$.

From (1), the interferometric coherence can be expressed approximately as the product of two terms, each of them related to a different source of decorrelation. The first term relates to the well-known spatial baseline decorrelation, which basically depends on the baseline distance and the relative frequency bandwidth of the system. The second term indicates a loss of coherence caused by the different heights of the scatterers inside the resolution cell, which is usually called volumetric effect.

Both terms in (1) depend on the baseline. However, as mentioned in Section I, there are other sources of loss of coherence that do not depend on the distance between the two antennas. One of them is the well-known temporal decorrelation, which is common in repeat-pass interferometric systems. Furthermore, even in single-pass interferometry, there can be some zones of the interferogram with degraded coherence due to a low SNR. The coherence degradation caused by the additive noise can be calculated as [20]

$$
\gamma=1 /\left(1+\mathrm{SNR}^{-1}\right)
$$

where the same SNR has been assumed in both images.

\section{B. Coherence Improvement by a Wavenumber Shift}

The spatial baseline decorrelation can be justified in the Fourier domain, because the difference between the two viewing angles produces a shift and a stretch of the imaged terrain spectra. Thus, the decorrelation is caused by the disjointed parts of the spectra. This concept is generally known as wavenumber shift [4]. This decorrelation can be eliminated by shifting the transmitted center frequency during the second measurement (Tuned Interferometric SAR) [21]. In this case, the equation that relates the transmitted center frequencies in both measurements is

$$
\frac{f_{02}}{f_{01}}=\frac{\sin \theta_{1}}{\sin \theta_{2}}
$$

If, as is usual in practical systems, it is not possible to change the transmitted frequency between the two acquisitions, one can also cancel the spatial baseline decorrelation by applying a common band prefiltering. Equation (1) can be rewritten by substituting the central frequencies of the resulting bands $f_{01}$ and $f_{02}$, yielding

$$
\begin{aligned}
\gamma \simeq & \operatorname{sinc}\left(\frac { 2 } { c } \Delta z \left[f_{01}\left(\sin \theta_{1} \cot \theta-\cos \theta_{1}\right)\right.\right. \\
& \left.\left.+f_{02}\left(\cos \theta_{2}-\sin \theta_{2} \cot \theta\right)\right]\right) .
\end{aligned}
$$

Once the first triangular term in (1) has been cancelled, the new coherence function is only dependent on the volumetric scattering effects. As explained above in the text, this kind of coherence degradation is caused by a change of the shape of the two imaged-terrain spectra. Thus, the spectral volume decorrelation cannot be eliminated by spectral filtering techniques, and other approaches must be used to reduce it. This volume decorrelation increases as the baseline distance does, but it is only important when the scatterers are distributed over a height range of many wavelengths. However, even in case of a short height distribution of the scatterers, the multiple reflections between them can amplify the volumetric scattering effects, and consequently, there is an extra loss of coherence [19], [21]. Moreover, since these multiple reflections, or shadowing phenomena between scatterers, are different for both antenna positions, the coherence degradation increases [22].

\section{Coherence Optimization by Polarimetry}

A possible way to reduce the spectral volume decorrelation is to make use of the entire scattering matrix (i.e., combining polarimetry and interferometry). When fully coherent polarimetric data are collected, the information associated with each pixel of the SAR image can be grouped into the following scattering vector [23]

$$
\underline{k}=\left[S_{\mathrm{hh}}, S_{\mathrm{hv}}, S_{\mathrm{vh}}, S_{\mathrm{vv}}\right]^{T}
$$

where ${ }^{T}$ indicates the matrix transposition operation, and $S_{p q}$ is the complex scattering coefficient for $q$-transmitted and $p$-received polarization, expressed in the orthogonal $(\mathrm{H}, \mathrm{V})$ linear basis. In backscatter, the vector reciprocity theorem forces the scattering matrix to be symmetric (i.e., $S_{\mathrm{hv}}=S_{\mathrm{vh}}$ ), and the scattering vector can be expressed as

$$
\underline{k}=\left[S_{\mathrm{hh}}, \sqrt{2} S_{\mathrm{hv}}, S_{\mathrm{vv}}\right]^{T} .
$$

As described in [23], another equivalent representation of the scattering vector, based on a Pauli matrices basis, is also commonly used in the literature. For our present purposes, both formulations are equivalent.

In interferometric measurements, the polarimetric information of both SAR images will be contained in two different scattering vectors $\underline{k}_{1}$ and $\underline{k}_{2}$ for image 1 and image 2, respectively. One can generate $3 \times 3$ possible interferograms using the interferometric phase calculated from all polarimetric $(\mathrm{H}, \mathrm{V})$ combinations with both images

$$
[\Phi]_{\text {int }}=\arg \left(\underline{k}_{1} \underline{k}_{2}^{* T}\right) .
$$


The interferometric coherence between all of these polarimetric combinations can be calculated from the following hermitian product matrices:

$$
\begin{aligned}
& {\left[T_{11}\right]=\left\langle\underline{k}_{1} \underline{k}_{1}^{* T}\right\rangle} \\
& {\left[T_{22}\right]=\left\langle\underline{k}_{2} \underline{k}_{2}^{* T}\right\rangle} \\
& {\left[\Omega_{12}\right]=\left\langle\underline{k}_{1} \underline{k}_{2}^{* T}\right\rangle}
\end{aligned}
$$

where $\left[T_{11}\right]$ and $\left[T_{22}\right]$ are the covariance matrices associated with the separate images, and $\left[\Omega_{12}\right]$ is a $3 \times 3$ complex matrix containing the interferometric information between polarimetric channels. Moreover, a new interferometric matrix $[\Gamma]$ can be defined as

$$
[\Gamma]=\left[\Omega_{12}\right] \cdot / \sqrt{\left[T_{11}\right] * *\left[T_{22}\right]}
$$

where the operators ./ and $* *$ indicate the element-by-element division and product operations. The interferometric coherence for every polarimetric combination in the $(\mathrm{H}, \mathrm{V})$ basis is the amplitude of the corresponding element of [Г], i.e.,

$$
|[\Gamma]|=\left[\begin{array}{lll}
\gamma_{\mathrm{hh}-\mathrm{hh}} & \gamma_{\mathrm{hh}-\mathrm{hv}} & \gamma_{\mathrm{hh}-\mathrm{vv}} \\
\gamma_{\mathrm{hv}-\mathrm{hh}} & \gamma_{\mathrm{hv}-\mathrm{hv}} & \gamma_{\mathrm{hv}-\mathrm{vv}} \\
\gamma_{\mathrm{vv}-\mathrm{hh}} & \gamma_{\mathrm{vv}-\mathrm{hv}} & \gamma_{\mathrm{vv}-\mathrm{vv}}
\end{array}\right]
$$

At this point, one can select the polarimetric combination with the maximum coherence in the $(\mathrm{H}, \mathrm{V})$ basis and therefore apply a first-level polarimetric optimization by extracting the interferometric phase associated with the corresponding element in the phase matrix $[\Phi]_{\text {int }}$.

Furthermore, the interferograms can be formed by using not only linear polarization states but also any other combination between arbitrary elliptical polarization states. All elliptical polarization states can be generated by applying a change of polarization basis to transform the scattering vector $k$ (expressed in the $(\mathrm{H}, \mathrm{V})$ basis) into another scattering vector $\underline{k}^{\prime}$, expressed in any other orthogonal basis $(\mathrm{X}, \mathrm{Y})[23]$

$$
\underline{k}^{\prime}=\left[S_{x x}, \sqrt{2} S_{x y}, S_{y y}\right]^{T}=\left[U_{3}\right] \underline{k}
$$

Physically, this transformation can be interpreted as a change of the selected scattering mechanisms in both images. Assuming that the scattering vector $\underline{k}$ is defined according to (6), the transformation matrix $\left[U_{3}\right]$ can be written as in (14), shown at the bottom of the page [24], where $\tau$ and $\phi$ are the ellipticity and orientation angles that define any polarization state. The $3 \times 3$ transformation matrix $\left[U_{3}\right]$ is special unitary. This constraint is required to ensure that the amplitude and phase of the wave are consistently defined as we change the polarization basis. After applying this change in the polarization basis, we can express the matrices defined in (8)-(10) in (15).

$$
\begin{aligned}
& {\left[T_{11}\right]_{(\tau, \phi)}=\left\langle\left[U_{3}\right] \underline{k}_{1} \underline{k}_{1}^{* T}\left[U_{3}\right]^{* T}\right\rangle=\left\langle\left[U_{3}\right]\left[T_{11}\right]\left[U_{3}\right]^{* T}\right\rangle} \\
& {\left[T_{22}\right]_{(\tau, \phi)}=\left\langle\left[U_{3}\right] \underline{k}_{2} \underline{k}_{2}^{* T}\left[U_{3}\right]^{* T}\right\rangle=\left\langle\left[U_{3}\right]\left[T_{22}\right]\left[U_{3}\right]^{* T}\right\rangle} \\
& {\left[\Omega_{12}\right]_{(\tau, \phi)}=\left\langle\left[U_{3}\right] \underline{k}_{1} \underline{k}_{2}^{* T}\left[U_{3}\right]^{* T}\right\rangle=\left\langle\left[U_{3}\right]\left[\Omega_{12}\right]\left[U_{3}\right]^{* T}\right\rangle .}
\end{aligned}
$$

The application of these polarimetric basis transformations allows us to form interferograms between all possible elliptical polarization states and combinations between both SAR images. The corresponding coherences are the moduli of the new $[\Gamma]_{(\tau, \phi)}$ matrix

$$
[\Gamma]_{(\tau, \phi)}=\left[\Omega_{12}\right]_{(\tau, \phi)} \cdot \sqrt{\left[T_{11}\right]_{(\tau, \phi)} *\left[T_{22}\right]_{(\tau, \phi)}}
$$

which are

$$
\left|[\Gamma]_{(\tau, \phi)}\right|=\left[\begin{array}{ccc}
\gamma_{x x-x x} & \gamma_{x x-x y} & \gamma_{x x-y y} \\
\gamma_{x y-x x} & \gamma_{x y-x y} & \gamma_{x y-y y} \\
\gamma_{y y-x x} & \gamma_{y y-x y} & \gamma_{y y-y y}
\end{array}\right]
$$

and the resulting interferometric phases are given by

$$
[\Phi]_{\mathrm{int}}=\arg \left([\Gamma]_{(\tau, \phi)}\right)
$$

where $x$ and $y$ denote two arbitrary elliptical polarizations. Again, the best interferometric phase estimate will be obtained by selecting the combination that maximizes the coherence between different polarization states in $\left|[\Gamma]_{(\tau, \phi)}\right|$. In general, two different transformation matrices $\left[U_{3}\right]$ should be used (one for each image) in order to combine all possible polarization states of image 1 with all in image 2 . That method cannot be implemented in a practical way, so the same transformation is applied to both images in the following. As will be described below, this simplification constitutes a limitation for comparing this approach with the exact formal solution of the optimization, and leads to suboptimum results.

We can further simplify the implementation of this approach by using some a priori knowledge about the values in $[\Gamma]_{(\tau, \phi)}$. The first assumption is that, in cases without temporal decorrelation and with a short baseline, the highest coherence will be given by the same polarization state in both images and therefore, the elements of the diagonal of the matrix $\left|[\Gamma]_{(\tau, \phi)}\right|$ will be much higher than the rest. Moreover, the definition of the geometrical angles $\tau$ and $\phi$ implies the symmetry

$$
\gamma_{x x-x x}(\tau, \phi)=\gamma_{y y-y y}(-\tau, \phi+\pi / 2)
$$

and, consequently, if the assumption is valid, we must calculate only the first and the second elements of the diagonal of $\left|[\Gamma]_{(\tau, \phi)}\right|$ and select the maximum one

$$
\gamma_{\text {opt }}=\max \left\{\gamma_{x x-x x}(\tau, \phi), \gamma_{x y-x y}(\tau, \phi)\right\}
$$

$$
\left[U_{3}\right]=\frac{1}{2}\left[\begin{array}{ccc}
\cos 2 \tau+\cos 2 \phi-j \sin 2 \phi \sin 2 \tau & \sqrt{2}(\sin 2 \phi+j \cos 2 \phi \sin 2 \tau) & \cos 2 \tau-\cos 2 \phi+j \sin 2 \phi \sin 2 \tau \\
j \sin 2 \tau-\sin 2 \phi \cos 2 \tau & \sqrt{2} j \cos 2 \phi \cos 2 \tau & \sin 2 \tau-\cos 2 \phi-j \sin 2 \phi \cos 2 \tau \\
j \sin 2 \tau+\sin 2 \phi \cos 2 \tau & \sqrt{2}(-\sin 2 \phi+j \cos 2 \phi \sin 2 \tau) & \cos 2 \tau+\cos 2 \phi+j \sin 2 \phi \sin 2 \tau
\end{array}\right] .
$$


By graphically representing the copolar and crosspolar interferometric coherences for every polarization state $\gamma_{x x-x x}(\tau, \phi)$ and $\gamma_{x y-x y}(\tau, \phi)$, it is possible to derive the existence of different independent scattering mechanisms inside the resolution cell. This approach is called polarization subspace method (PSM), because in the maximization of the coherence, we are not using all the polarimetric information. We are only considering the copolar and crosspolar elements, and the same polarization basis transformation is assumed in both images. In the case of a single dominant scattering behavior, both coherence functions generally present only one absolute maximum. Thus, by selecting the highest one, we will find the best polarization subspace that can be used to generate the optimum DEM of the scene. On the other hand, in the case of vegetated surfaces, it is possible to identify various local coherence maxima. These are related to different independent scattering mechanisms that lead to the "locally best" height estimates. Hence, the polarization states corresponding to these maxima can be used to calculate the height of different layers into which the vegetation target can be decomposed.

The PSM has an important limitation. In those practical cases where there exists a large amount of temporal decorrelation or the difference between the two viewing angles (baseline) is large, the scattering behavior of the observed terrain changes between the two acquisitions and therefore, the maximum coherence is reached by different polarization subspaces in both images. It means that we would need to correlate the backscattering field obtained by applying different polarization basis transformations to both SAR images, and consequently, the described optimization process would become too computationally intensive. Therefore, instead of using the PSM, it would be useful to find a mathematical expression to calculate directly the optimum polarization states that maximize the interferometric coherence.

This optimization problem has been solved in [9] by introducing a vector formulation into the interferometric coherence definition. For doing that, two complex weight unitary vectors $\underline{w}_{1}$ and $\underline{w}_{2}$ can be defined to compute the following complex scalars $\mu_{1}$ and $\mu_{2}$ :

$$
\mu_{1}=\underline{w}_{1}^{* T} \underline{k}_{1} \quad \mu_{2}=\underline{w}_{2}^{* T} \underline{k}_{2} .
$$

These two complex scalars are the projection of the scattering vectors $\underline{k}_{1}$ and $\underline{k}_{2}$ onto the vectors $\underline{w}_{1}$ and $\underline{w}_{2}$. Thus, the scalars $\mu_{1}$ and $\mu_{2}$ can be interpreted as the complex scattering coefficients for the scattering mechanisms $\underline{w}_{1}$ and $\underline{w}_{2}$ [9]. This notation allows us to express the interferometric coherence as follows:

$$
\gamma=\frac{\left|\left\langle\mu_{1} \mu_{2}^{*}\right\rangle\right|}{\sqrt{\left\langle\mu_{1} \mu_{1}^{*}\right\rangle\left\langle\mu_{2} \mu_{2}^{*}\right\rangle}}=\frac{\left|\left\langle\underline{w}_{1}^{* T}\left[\Omega_{12}\right] \underline{w}_{2}\right\rangle\right|}{\sqrt{\left\langle\underline{w}_{1}^{* T}\left[T_{11}\right] \underline{w}_{1}\right\rangle\left\langle\underline{w}_{2}^{* T}\left[T_{22}\right] \underline{w}_{2}\right\rangle}} .
$$

The objective of the optimization algorithm is to select those scattering mechanisms $\underline{w}_{1}$ and $\underline{w}_{2}$ that maximize the interferometric coherence defined in (22). The optimization problem leads to the following equations:

$$
\begin{aligned}
& {\left[T_{11}\right]^{-1}\left[\Omega_{12}\right]\left[T_{22}\right]^{-1}\left[\Omega_{12}\right]^{* T} \underline{w}_{1}=\nu \underline{w}_{1}} \\
& {\left[T_{22}\right]^{-1}\left[\Omega_{12}\right]^{* T}\left[T_{11}\right]^{-1}\left[\Omega_{12}\right] \underline{w}_{2}=\nu \underline{w}_{2} .}
\end{aligned}
$$
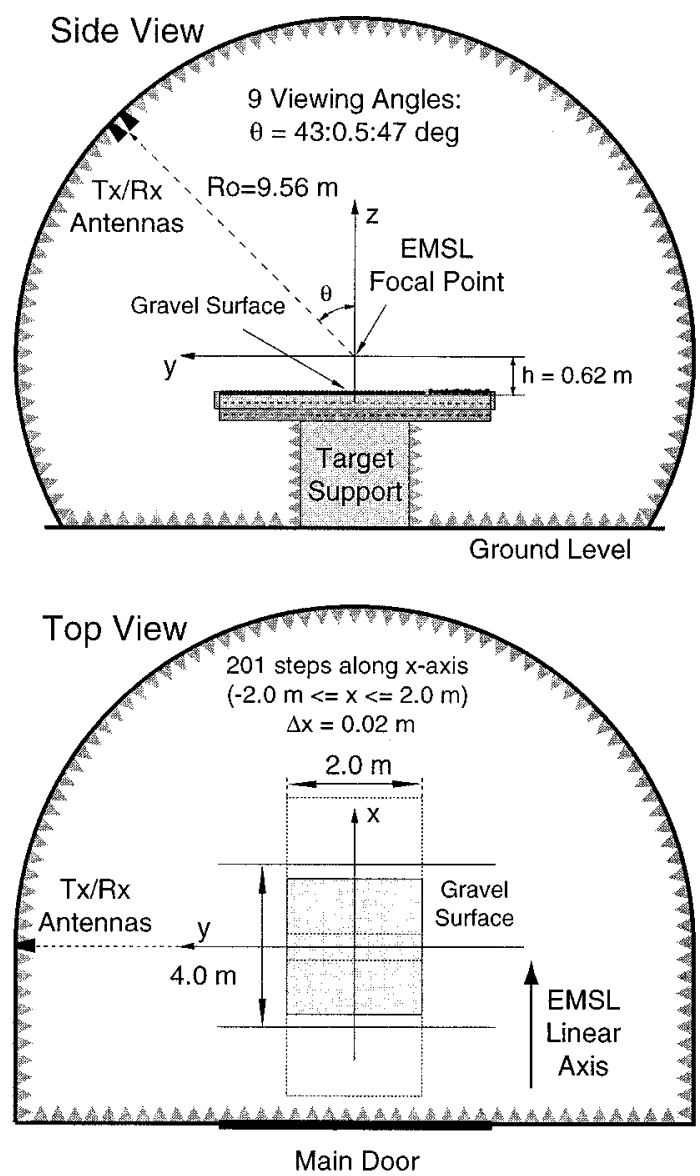

Fig. 2. Experimental set-up for the DEM generation of the gravel surface.

Equation (23) corresponds to two $3 \times 3$ complex eigenvalue problems for the vectors $\underline{w}_{1}$ and $\underline{w}_{2}$. The eigenvalues are real, and the optimum coherence will be given by the highest one. The solution is those optimum scattering mechanisms that minimize the interferometric decorrelation. Unlike the maximization method proposed in (20), the solution of the polarimetric optimization problem formulated in (23) takes into consideration that the optimum coherence can be given by different scattering mechanisms $\left(\underline{w}_{1_{\mathrm{opt}}} \neq \underline{w}_{2_{\mathrm{opt}}}\right)$ in each SAR image, because the scattering behavior of the observed surface may change.

Having found the optimum scattering mechanisms $\underline{w}_{1_{\mathrm{opt}}}$ and $\underline{w}_{2_{\text {opt }}}$, the interferometric phase will be given by:

$$
\phi_{\text {int }}=\arg \left(\mu_{1} \mu_{2}^{*}\right)=\arg \left(\underline{w}_{1_{\text {opt }}}^{* T} \underline{k}_{1} \underline{k}_{2}^{* T} \underline{w}_{2_{\text {opt }}}\right) .
$$

As outlined in the introduction, the interferometric phase associated with the first pair of optimum scattering mechanisms has been used in the experiments to generate the DEM of nonvegetated surfaces, and the second and third pair of optimum scattering mechanisms have been employed in addition to the first in vegetation height estimations.

\section{EXPERIMENTS ON DEM GENERATION OF NONVEGETATED NATURAL SURFACES}

In the first experiment, a $2 \times 2 \mathrm{~m}$ planar rough surface of gravel has been measured. A series of fully polarimetric strip-map SAR data sets have been acquired in the frequency 


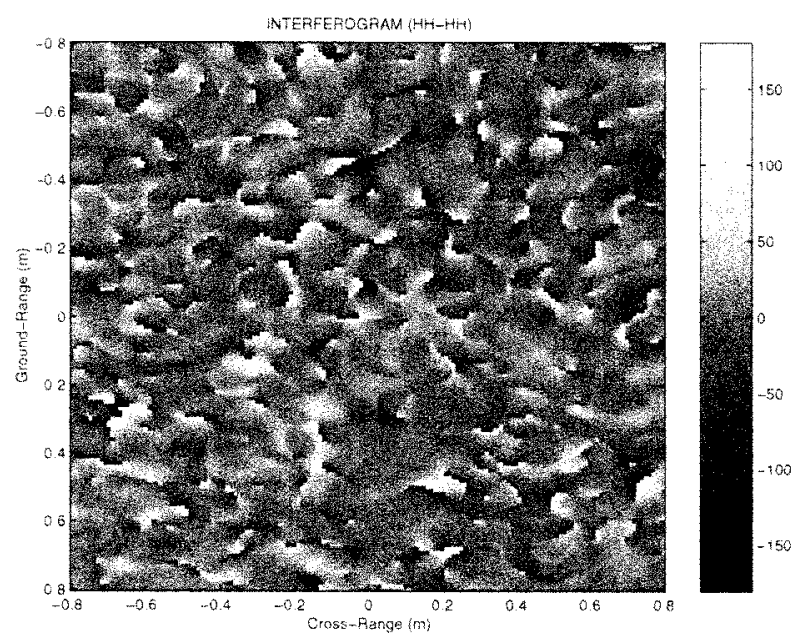

(a)

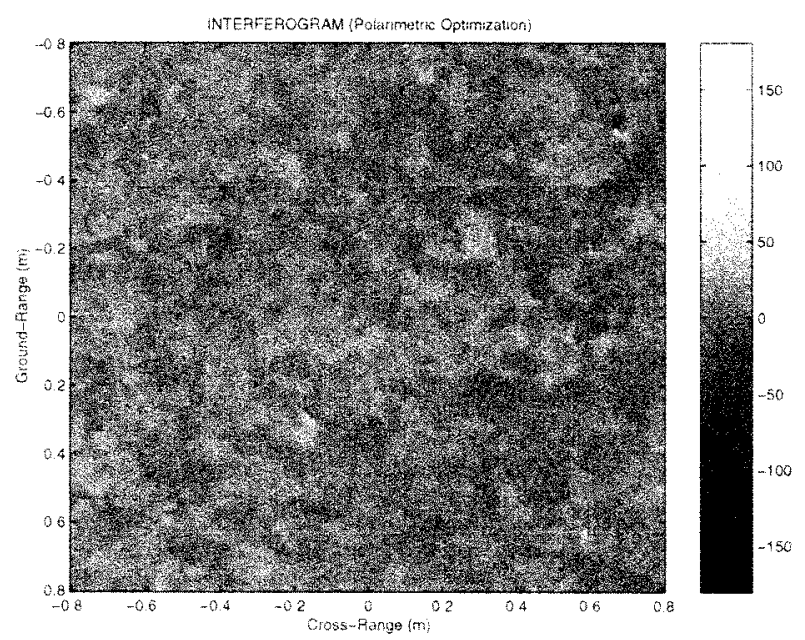

(c)

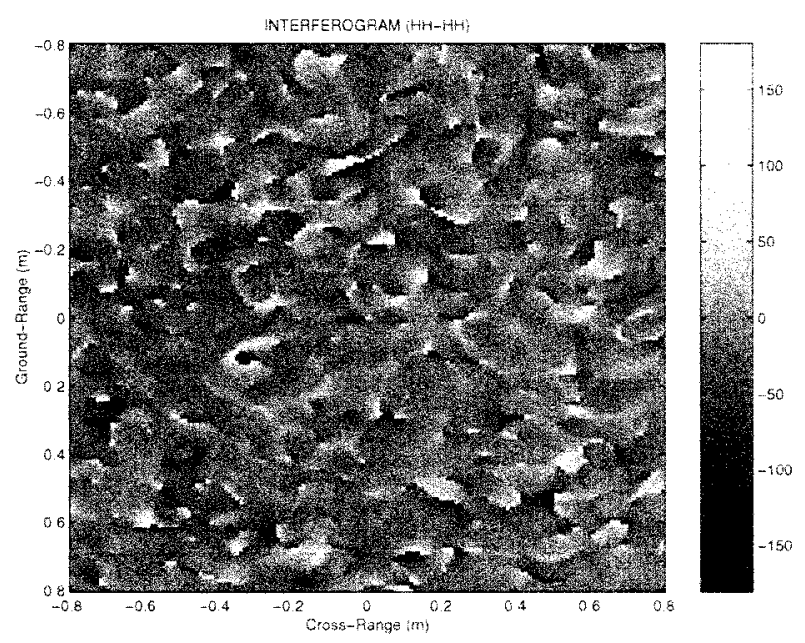

(b)

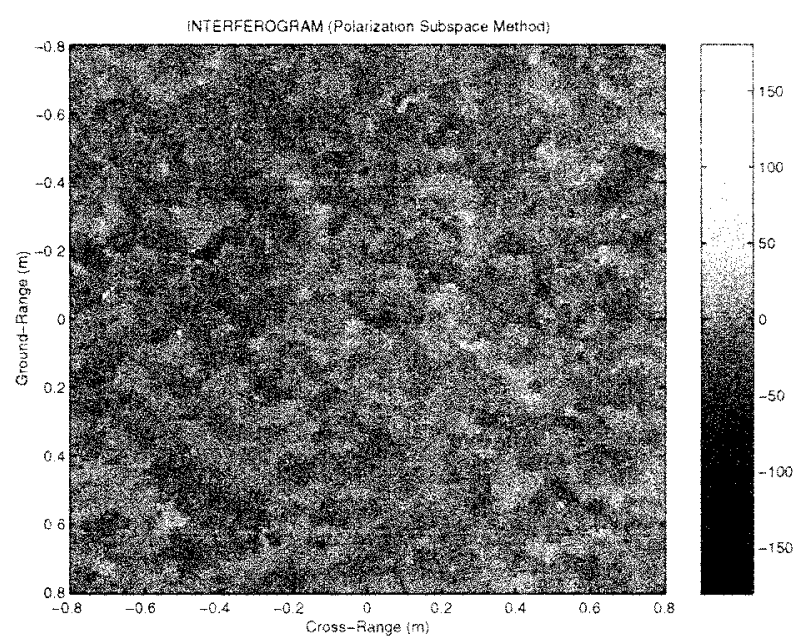

(d)

Fig. 3. Interferograms for a planar rough surface of gravel using a baseline $B=1^{\circ}$. (a) Interferogram formed by the two HH images, (b) obtained by removing the disjoint parts of the spectra, (c) obtained by applying the polarimetric optimization algorithm, and (d) obtained by applying the polarization subspace method.

range $8-12 \mathrm{GHz}$, with nine uniformly spaced viewing angles ranging from $43^{\circ}$ to $47^{\circ}$ (thus having angular baselines from $0.5^{\circ}$ up to $4^{\circ}$ ). The experimental set-up of the measurement is shown in Fig. 2. With such a measurement geometry, the near-field phase distortion can be accounted for by considering a spherical wavefront illuminating the target. The 2-D complex reflectivity images for each polarization have been reconstructed on a horizontal plane using a near-field SAR processor especially suited for the EMSL geometry [25]. The synthetic aperture length is $4 \mathrm{~m}$, and the cross-range and ground-range resolutions are approximately $4 \mathrm{~cm}$.

It has been proved experimentally that the penetration depth of electromagnetic waves at $\mathrm{X}$ band into this kind of terrain is larger than its actual depth $(\approx 2 \mathrm{~cm})$. Therefore, this target is in fact a nonvegetated volume scatterer and consequently, the volume decorrelation must be taken into account. Although the height distribution of the scatterers is lower than the wavelength, the discrete physical constitution of the target causes some multiple reflections between scatterers that amplify the volumetric effects and produce an important loss of coherence that is not considered in (1). Fig. 3 shows the interferograms corresponding to this flat gravel surface for a baseline $B=1^{\circ}$. The expected interferometric phase should stay around zero, because the surface does not present any slope. However, the interferogram shown in Fig. 3(a), which has been generated using two SAR images with $\mathrm{HH}$ polarization, exhibits some zones where the phase level is very high. This phase error is mainly due to the spatial baseline decorrelation and the volumetric scattering effects. There are also some noisy zones that correspond to those zones of the SAR images with very low amplitude (close to zero). In those areas, the system thermal noise is dominant, and the resulting interferometric phase is noisy. If we apply a common spectral band filter to both spectra as suggested in Section II-B, the spatial baseline decorrelation is eliminated, and the quality of the obtained interferogram is improved (see Fig. 3(b) and the comparison in Fig. 5). Nevertheless, there are still some 


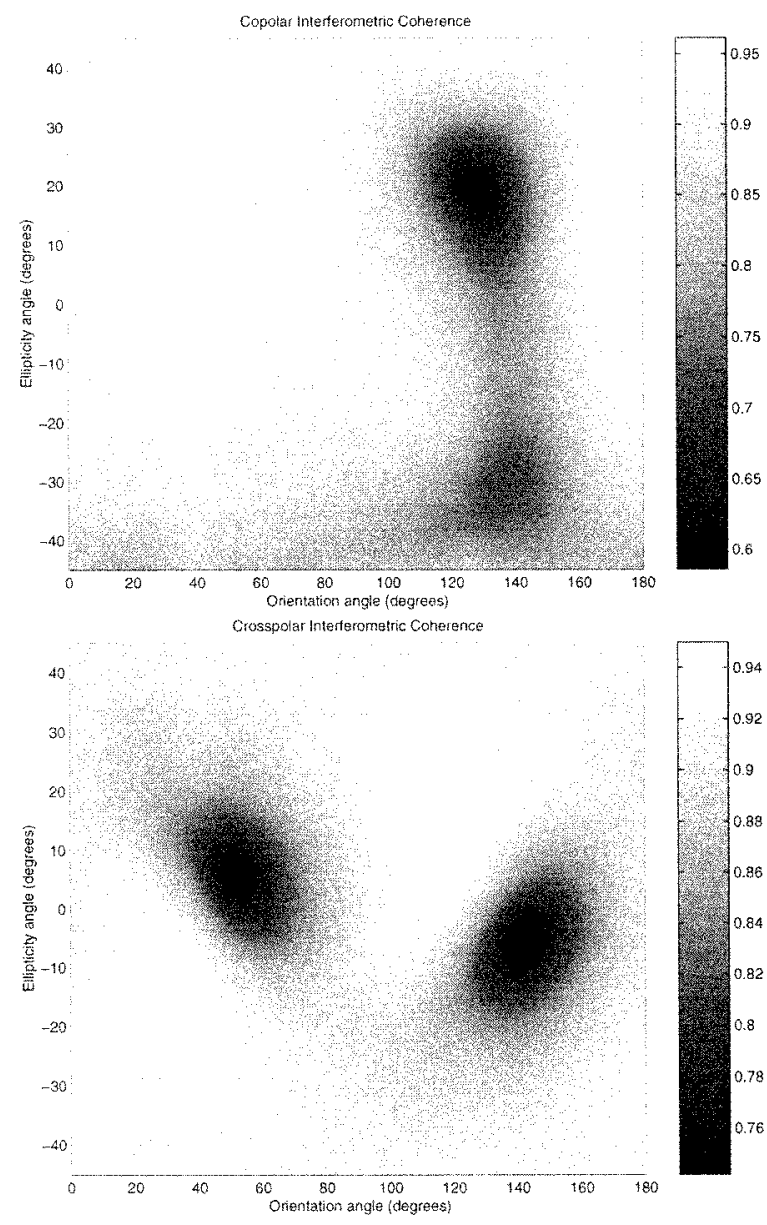

Fig. 4. Coherence maps for all $(\tau, \phi)$ polarization states, corresponding to a planar rough surface of gravel using a baseline $B=1^{\circ}$. Top: Copolar $\gamma_{x x-x x}(\tau, \phi)$. Bottom: Crosspolar $\gamma_{x y-x y}(\tau, \phi)$.

zones with a high phase error due to the volume scattering effects and the influence of the system noise. Fig. 3(c) shows the interferogram obtained after applying the polarimetric coherence optimization algorithm formulated in Section II-C. It is now evident that the noisy zones have been totally removed, and the interferogram presents a constant phase level near zero, which corresponds to the actual profile of the surface.

For illustrating how the polarimetric optimization works, the PSM has been also applied to these data. Fig. 3(d) shows the interferogram obtained by applying this approach. The copolar $\gamma_{x x-x x}$ and crosspolar $\gamma_{x y-x y}$ interferometric coherences obtained for every polarization state $(\tau, \phi)$ are represented in Fig. 4. These coherence maps correspond to a $25 \times 25 \mathrm{~cm}$ square zone located at the center of the planar rough surface of gravel. The copolar coherence exhibits only one maximum at $\left(\tau=26^{\circ}, \phi=20^{\circ}\right)$. In the crosspolar case, since the backscattering matrix is symmetric $\left(S_{\mathrm{hv}}=S_{\mathrm{vh}}\right)$, the coherence signature shows an uneven symmetry $\gamma_{x y-x y}(\tau, \phi)=\gamma_{x y-x y}(-\tau, \phi+\pi / 2)$ and thus, the two maxima that we can identify correspond to the same scattering mechanism $\left(\tau=-19^{\circ}, \phi=26^{\circ}\right)$. Therefore, we can state that each coherence function shows a single absolute maximum, which means that the measured scene presents only

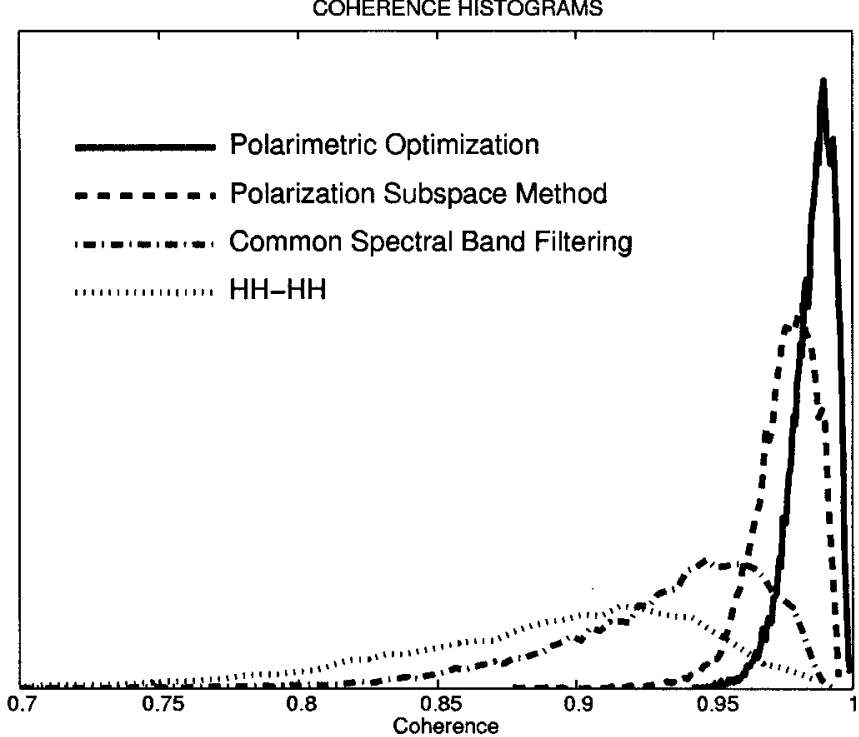

(a)

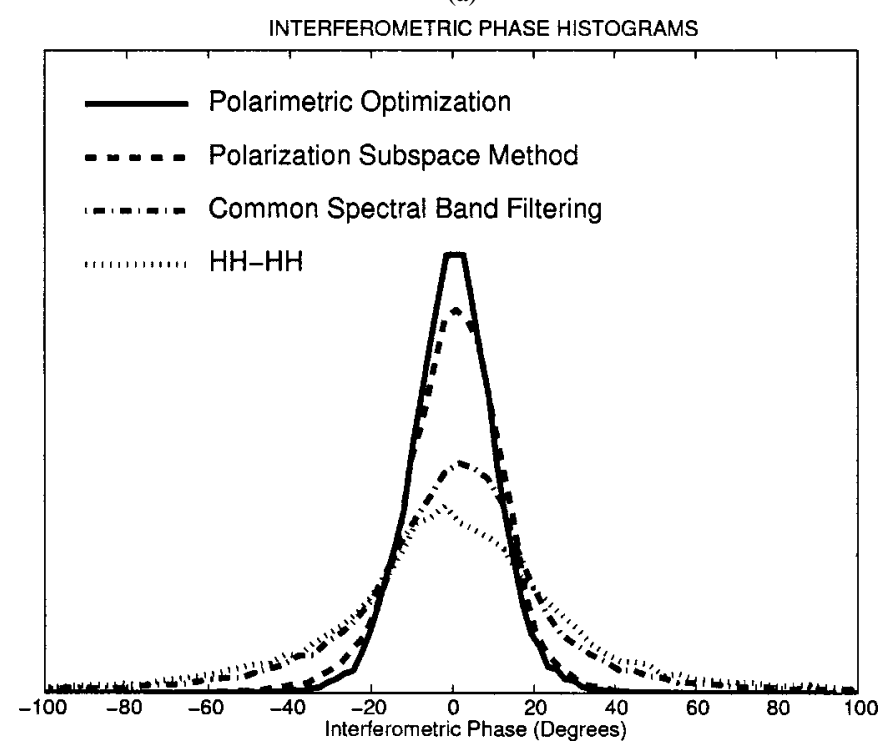

(b)

Fig. 5. (a) Interferometric coherence and (b) phase histograms for a planar rough surface of gravel using a baseline $B=1^{\circ}$. Comparison between the results obtained from the two $\mathrm{HH}$ images (dotted), the images obtained after removing the disjoint parts of the spectra (dash-dotted), the images resulting form the polarization subspace method (dashed), and the images resulting from the polarimetric optimization algorithm (solid).

one dominant scattering mechanism. According to the PSM, the optimum interferogram would be formed by combining those elements of the backscattering vectors expressed in that polarization subspace $(\tau, \phi)$ and combination (copolar or crosspolar), where the coherence is maximum. In this particular case, the maximum coherence is given by the polarization state $\left(\tau=26^{\circ}, \phi=20^{\circ}\right)$ of the copolar coherence function $\gamma_{x x-x x}$. This means that the optimum interferometric phase corresponding to this zone of the surface will be formed by the complex combination $\arg \left(S_{x x 1} S_{x x 2}^{*}\right)$, where $S_{x x}$ denotes the first element of the scattering vector of each pixel of both images expressed into the optimum polarization state $\left(\tau=26^{\circ}, \phi=20^{\circ}\right)$. As we can see in Fig. 4, there are some polarization states in which the coherence falls to very low 
TABLE I

CoHerence And Height ERror for the Planar Surface $\left(B=1^{\circ}\right)$

\begin{tabular}{l|c|c|c}
\hline & Mean Coherence & Phase Std. Dev. & Height Std. Dev. \\
\hline HH-HH & 0.8851 & $41.45^{\circ}$ & $7.27 \mathrm{~cm}$ \\
Spectral Common Band Filtering & 0.9293 & $33.70^{\circ}$ & $5.92 \mathrm{~cm}$ \\
PSM & 0.9755 & $12.59^{\circ}$ & $2.21 \mathrm{~cm}$ \\
Polarimetric Optimization & 0.9850 & $10.82^{\circ}$ & $1.90 \mathrm{~cm}$ \\
\hline
\end{tabular}

values (close to 0.7 and 0.6 ). These low coherence values are produced by nulls of the copolar and crosspolar backscatter signatures of the target. Those polarizations with low backscatter return lead to coherences that are influenced by the SNR.

The comparison between these results has been performed on the basis of their statistics. Fig. 5 illustrates the coherence and interferometric phase histograms corresponding to the four interferograms shown in Fig. 3, and Table I presents the mean coherences and the corresponding phase and height standard deviations. As we can see, the coherence value measured in the HH basis is lower than that expected from (1) and (4), even if the height of the volume scatterer is taken into account. This additional degradation in the coherence can be caused by multiple reflections or shadowing phenomena between scatterers, when these effects are different for both antenna positions [19], [22]. A polarimetric decomposition carried out on this target has proven that its average scattering mechanism exhibits high entropy and is dominated by multiple scattering. Moreover, the type of scattering mechanism (described by the mean alpha angle [6]) changes between the two interferometric acquisitions. After applying the optimization algorithm, the interferometric coherence is close to one, and the resulting height error has been drastically reduced. As shown in Table I, the coherence obtained with the polarimetric optimization algorithm is higher than that obtained by selecting the polarization state that maximizes the coherence maps (PSM). In fact, this result was expected, because the scattering mechanisms ( $\underline{w}_{1_{\mathrm{opt}}}$ and $\left.\underline{w}_{2_{\text {opt }}}\right)$ obtained by solving the optimization problem correspond to linear combinations of polarization states that can be different for both images $\left(\underline{w}_{1_{\text {opt }}} \neq \underline{w}_{2_{\text {opt }}}\right)$ in contrast to the PSM, where the same polarization state is employed in both images.

We have also proved experimentally that these conclusions can be generalized for different baselines. Fig. 6 presents the interferometric coherence obtained after applying the proposed coherence optimization techniques for various baselines. For scalar interferometry, there is an important degradation of the interferometric coherence as the baseline increases, but after applying the polarimetric optimization, the coherence gets close to one for all baselines. It means that it is possible to use longer baselines in order to improve the height accuracy while keeping the coherence high. It can also be noted that the results obtained by solving the polarimetric optimization are better than those obtained with the PSM as the baseline increases. Again, the reason is that the scattering behavior of the observed surface changes substantially as the difference between the two incidence angles increases and consequently, the optimum coherence can be given by applying different polarization basis transformations to each SAR image.

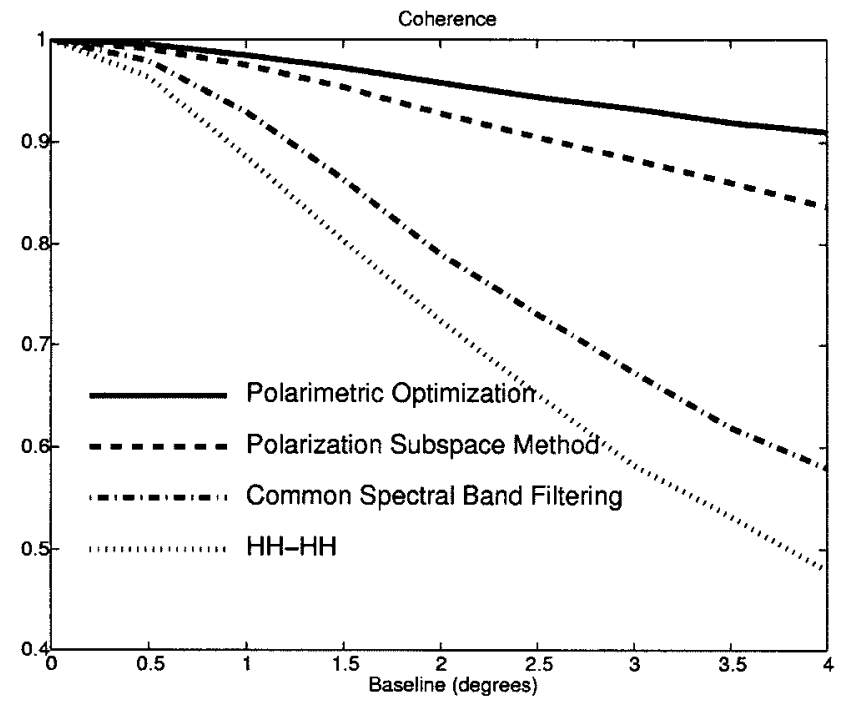

Fig. 6. Interferometric coherence as a function of the baseline for a planar rough surface of gravel. Comparison between the two HH images (dotted), obtained after removing the disjointed parts of the spectra (dash-dotted), which were obtained from the subspace method (dashed), and the images resulting from the polarimetric optimization algorithm (solid).

\section{EXPERIMENTS ON VEGETATION HEIGHT Estimation}

Three vegetation samples with different morphological structures (see photographs on Fig. 7) were measured in the following experiments.

1) Maize sample: a stand of $6 \times 6$ young plants of maize about $1.8 \mathrm{~m}$ high, uniformly planted in a square container with side length $2 \mathrm{~m}$. The plants show a green vertical fresh trunk with a diameter of about $4 \mathrm{~cm}$. The stems carry wide leaves from a height of $40 \mathrm{~cm}$ up to the top. The leaves are about $30-40 \mathrm{~cm}$ long and $7-8 \mathrm{~cm}$ wide and are oriented at around $45^{\circ}$ with respect to the trunk.

2) Cluster of young fir trees: a stand of nine young fir trees about $1.8 \mathrm{~m}$ high, regularly planted in a round container with diameter $2.4 \mathrm{~m}$. The trees are very close together. The structure of each tree is cone-shaped, with no branches in the upper $20 \mathrm{~cm}$. The branches bear 2-3 $\mathrm{cm}$ needles showing a brush-like distribution.

3) Rice sample: a stand of $9 \times 9$ rice plants about $0.6 \mathrm{~m}$ high, uniformly distributed in a square container with side length $1 \mathrm{~m}$. Each plant presents a cluster of green stems or long leaves that originates directly from the ground. In the upper half of the sample, these stems are notably bent and oriented in a random fashion. The leaves are about $2 \mathrm{~cm}$ wide. The soil was flooded to replicate the natural conditions of rice crops.

Note that, in contrast to other measurements previously performed at the EMSL, the ground under the plants was not covered by microwave absorbers. In this way, the ground interaction with the plants, which plays a key role in the backscatter response and is crucial to the height estimation algorithm, is present on the data.

The set-up employed in this case is different from that used in conventional SAR interferometry, since the whole vegetation sample must be confined inside a single resolution cell in order 

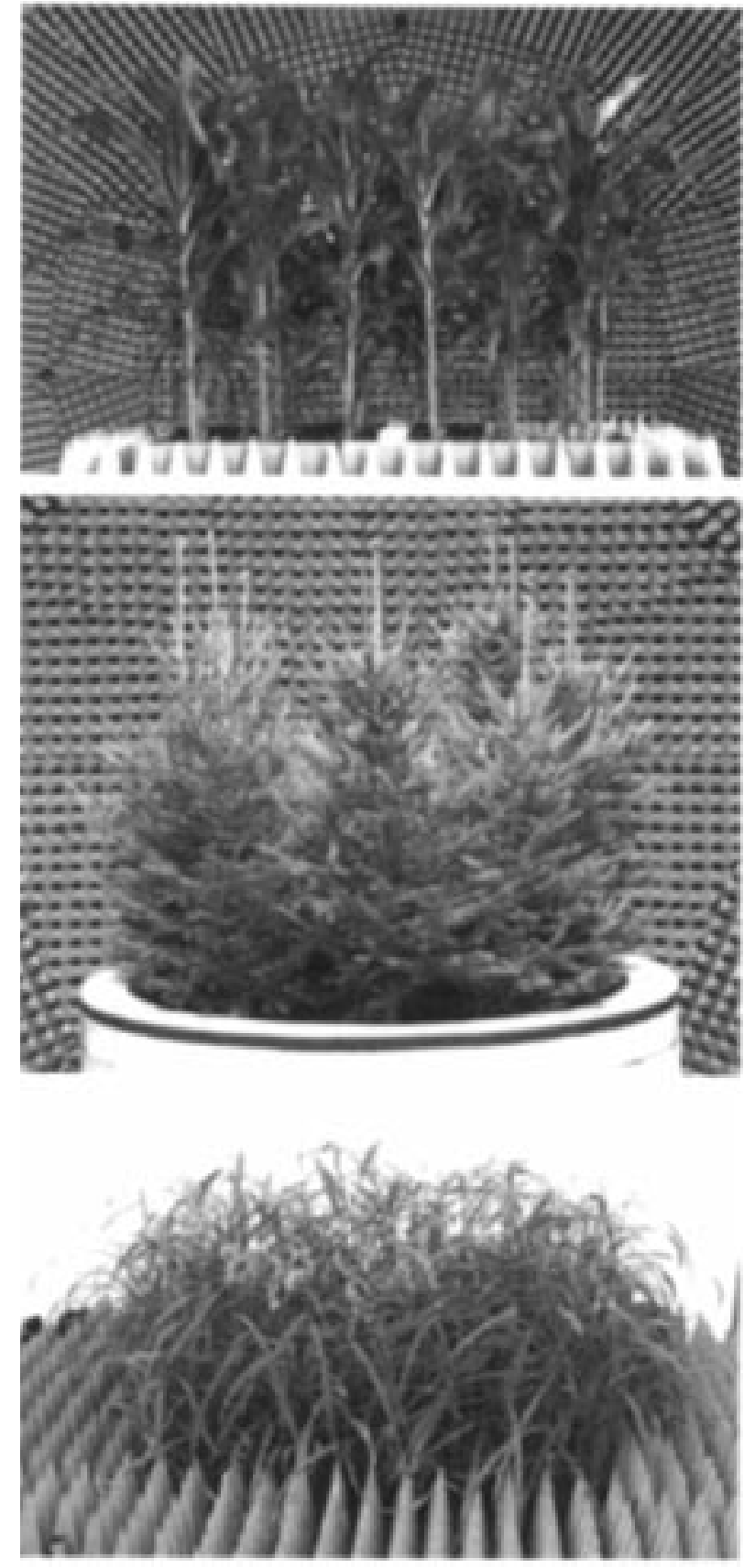

Fig. 7. Photographs of the vegetation samples: maize (top), cluster of young fir trees (center), and rice (bottom).

to apply the algorithm and, as a result, there is only one pixel in each image. Consequently, spatial averaging cannot be performed. Instead, two alternative averaging techniques have been used. The first consists in rotating the target in azimuth in order to obtain independent samples. The selected angular step depends on the working frequency and the target size. The second is known as frequency averaging or frequency agility and is based on collecting the radar returns from nonoverlapping frequencies. Both techniques are equivalent to some extent, as explained in [26].

Two kinds of scanning geometries have been employed. The first consists in measuring the backscattering at a few closely-spaced incidence angles, and rotating the sample in azimuth over $360^{\circ}$ in order to obtain as many independent samples as possible. The second type of measurements were originally planned for three-dimensional inverse SAR (ISAR) imaging. Its particular characteristic is that the target is rotated only about a narrow angular span (typically $10^{\circ}$ to $20^{\circ}$ ), and it employs a very small step as is usually needed in radar imaging. Consequently, the number of independent samples in azimuth is low for our purposes, and additional frequency averaging must be applied to reduce the variability of the results.

To minimize the phase noise and hence improve the accuracy of the height estimates, the interferograms for each scattering mechanism were multilooked over the entire set of independent samples. The number of samples, which can be regarded as pixels of a conventional image, changed between experiments, as was explained above. Note that we are not affected by the loss of resolution that the multilook procedure entails because, our scene is completely homogeneous (in fact, we are always looking at the same target). The conversion from interferometric phase to height is calculated by using typical interferometric equations with the same formulation as [9]. It is important to emphasize that the estimates presented in the next results are not affected by phase ambiguities, because the maximum height of the plants does not exceed the ambiguity height.

Note that the algorithm yields three phases associated with three scattering mechanisms at different heights. Then, three height estimates are computed as the height differences between the three possible combinations of scattering mechanisms (i.e., first-second, first-third, and second-third). Since there is no $a$ priori information about the relative height levels of those three mechanisms, the final inverted height is calculated as the maximum of the three differences.

As explained in the introduction, one of the main advantages of this technique is that no DEM, or knowledge about the ground level, is required for obtaining the height of the target. It is important to make clear that the decomposition into different scattering mechanisms resulting from the coherence optimization can be performed, although some dominant scatterers exhibit a response much stronger than the others. This is because the decomposition is not based on the backscatter values but on interferometry. The maximum difference in backscatter between dominant and secondary scatterers that would mask the latter is still under investigation.

The first experiment was carried out on the maize sample. The incidence angle ranged from $44^{\circ}$ to $45^{\circ}$, with a step of $0.25^{\circ}$, and the target was rotated in azimuth with a step of $5^{\circ}$, thus obtaining 72 independent samples. The frequency span was 0.7 to $9.5 \mathrm{GHz}$. The reflectivity images were computed with a narrow bandwidth $(40 \mathrm{MHz})$ in order to enclose the whole target inside the resolution cell, and the wavenumber shift was applied to avoid the dependence on ground-range, as suggested in [9]. The height estimation results as a function of the frequency are shown in Fig. 8 for baselines of $0.25^{\circ}$ and $0.5^{\circ}$. The displayed frequency range is limited to L-band, S-band, and C-band because at shorter wavelengths, some ambiguities arise in the results due to the large baselines that have been used. For each frequency, the plotted result is averaged over all combinations of incidence angles with the chosen baseline (i.e., four for $0.25^{\circ}$ and three for $0.5^{\circ}$ ). 

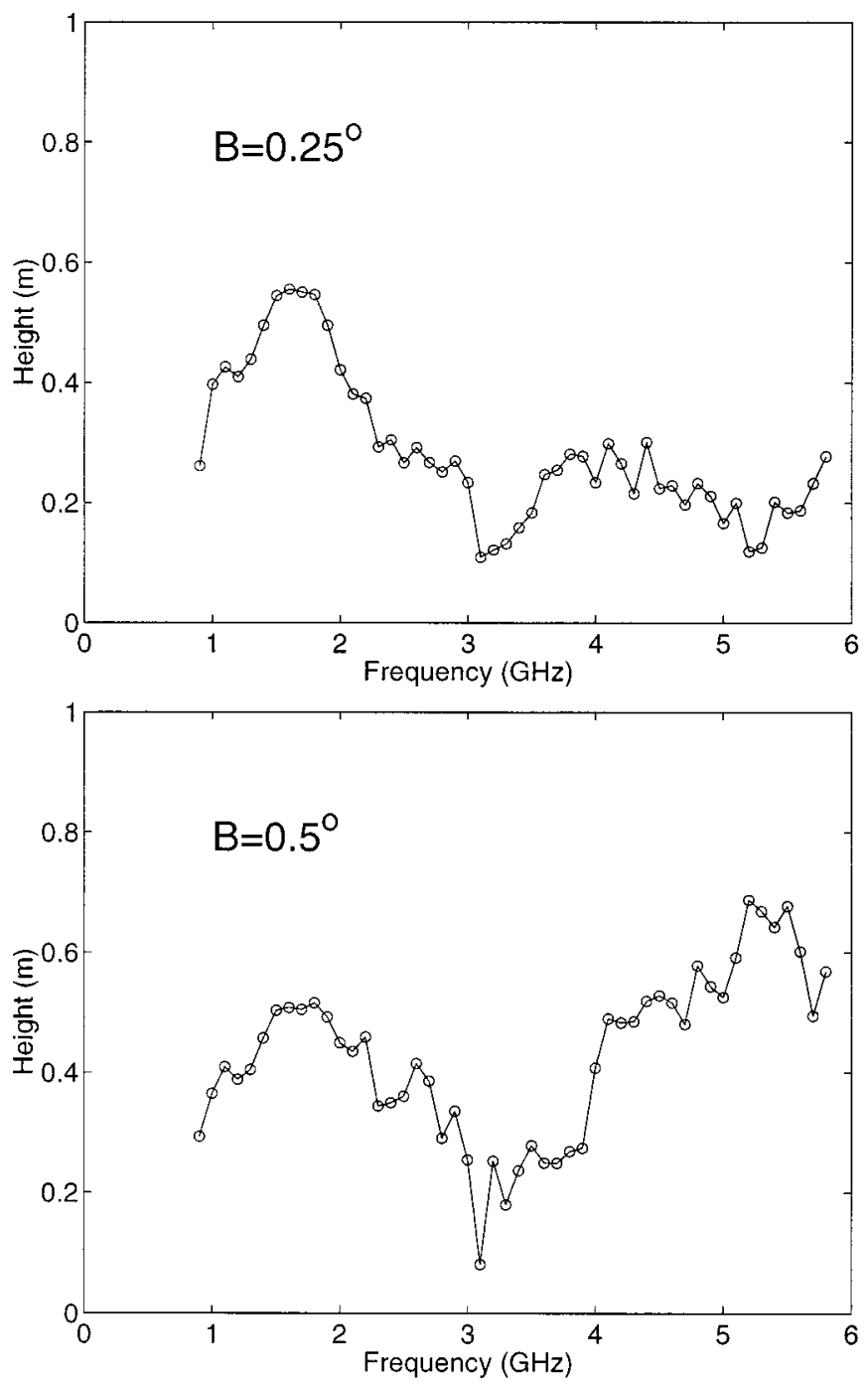

Fig. 8. Height estimates versus frequency for the maize sample at L-band, S-band, and C-band. Top: $B=0.25^{\circ}$. Bottom: $B=0.5^{\circ}$.

It is interesting to test the performance of the algorithm as a function of frequency and baseline. This is an important feature that can only be studied with facilities like the EMSL. In the case of maize, the penetration is quite high at all bands in the microwave region (even at $\mathrm{X}$ band), and the plants are short enough for the radar to be able to penetrate down to the ground, which is of crucial importance for retrieving the true height of the sample. The estimates shown in Fig. 8 present quite a complicated dependence on the working frequency. An inspection of the estimation procedure gives us some possible reasons for these varying estimates. Any change in the estimates is produced by: 1) a displacement (absolute or relative to the other mechanisms) of the phase center of a mechanism when the frequency changes, and/or 2) an interchange in the physical meaning of the scattering mechanism, which can produce a swap in their relative order. In general, the height estimates are higher for the baseline of $0.5^{\circ}$ and mainly at high frequencies. There is a band around 3-4 GHz, where the estimates change their trend for both baselines. This phenomenon may be caused by a change in the order of the optimum scattering mechanisms. The rest of the fluctu- ations are due to variations in the height level of the individual mechanisms. Although not presented here because of space constraints, this justification has been proven by plotting the height values of the individual mechanisms.

The justification of these estimates is the subject of an ongoing research. Anyway, some preliminary hints can be derived from a physical model of the target. At these angles of incidence and frequencies, the dominant scattering mechanism is expected to be the stem-ground interaction. The phase center of this mechanism has been demonstrated to be placed ideally at ground level [27]. A secondary scattering mechanism, usually weaker than the stem-ground interaction, is the direct backscatter from the stems and leaves [28]. However, the position of the effective phase center of this secondary mechanism is not well defined, and we can only say that it should be somewhere between the top and the bottom of the plants. A precise model should also include important concepts such as the attenuation in propagating through the medium, the different extinction coefficients that different polarizations may present, etc. Another key issue in this retrieval algorithm is the physical meaning of the scattering mechanisms derived from the coherence optimization. These mechanisms are calculated for optimizing the coherence, but nothing is said about their actual position inside the samples. As part of the ongoing research, this selection of scattering mechanisms is being compared to others, based on an a priori knowledge of the distribution of scattering properties of the target (for example, selecting $\mathrm{HH}$ and $\mathrm{HV}$ polarizations as in [29]). Note again that a complete justification of the retrieved values is out of the scope of the present paper.

The application of the PSM to vegetation height retrieval is briefly illustrated in the following. Fig.9 shows the obtained copolar $\gamma_{x x-x x}$ and crosspolar $\gamma_{x y-x y}$ coherences as a function of every polarization state $(\tau, \phi)$. These coherence maps correspond to the maize sample at $3.5 \mathrm{GHz}$ for a baseline of $0.25^{\circ}$. In this case, the copolar coherence signature presents two different local maxima $\left(\tau=-19^{\circ}, \phi=132^{\circ}\right)$ and $\left(\tau=37^{\circ}, \phi=\right.$ $\left.164^{\circ}\right)$, whereas the crosspolar case exhibits one maximum at ( $\tau=-8^{\circ}, \phi=3^{\circ}$ ), which in the plot is repeated due to the symmetries commented in Section II. Fig. 10 shows the height values obtained for every polarization state $(\tau, \phi)$ corresponding to those copolar and crosspolar coherence functions presented in Fig. 9. The final estimate is derived by extracting the maximum height difference between these three polarization states. For this particular frequency, the estimated height is $0.4 \mathrm{~m}$.

Table II shows the height estimates for the maize sample at three different frequencies (L-band, S-band, and C-band) and two different baselines $\left(0.25^{\circ}\right.$ and $\left.0.5^{\circ}\right)$. In most cases, the estimates are quite dissimilar. An interpretation of these discrepancies has not been found yet.

The second experiment dealt with the cluster of young fir trees. The measurement was performed from 1 to $10 \mathrm{GHz}$, with 25 elevation incidence angles ranging from $39^{\circ}$ to $51^{\circ}$ and a step of $0.5^{\circ}$. As before, the bandwidth used in the image reconstruction was $40 \mathrm{MHz}$. This measurement corresponds to an ISAR imaging experiment and therefore, the rotation in azimuth did not cover the whole circle. There were two separate azimuth spans of $12^{\circ}$ with a step of $0.5^{\circ}$. By looking at independence cri- 


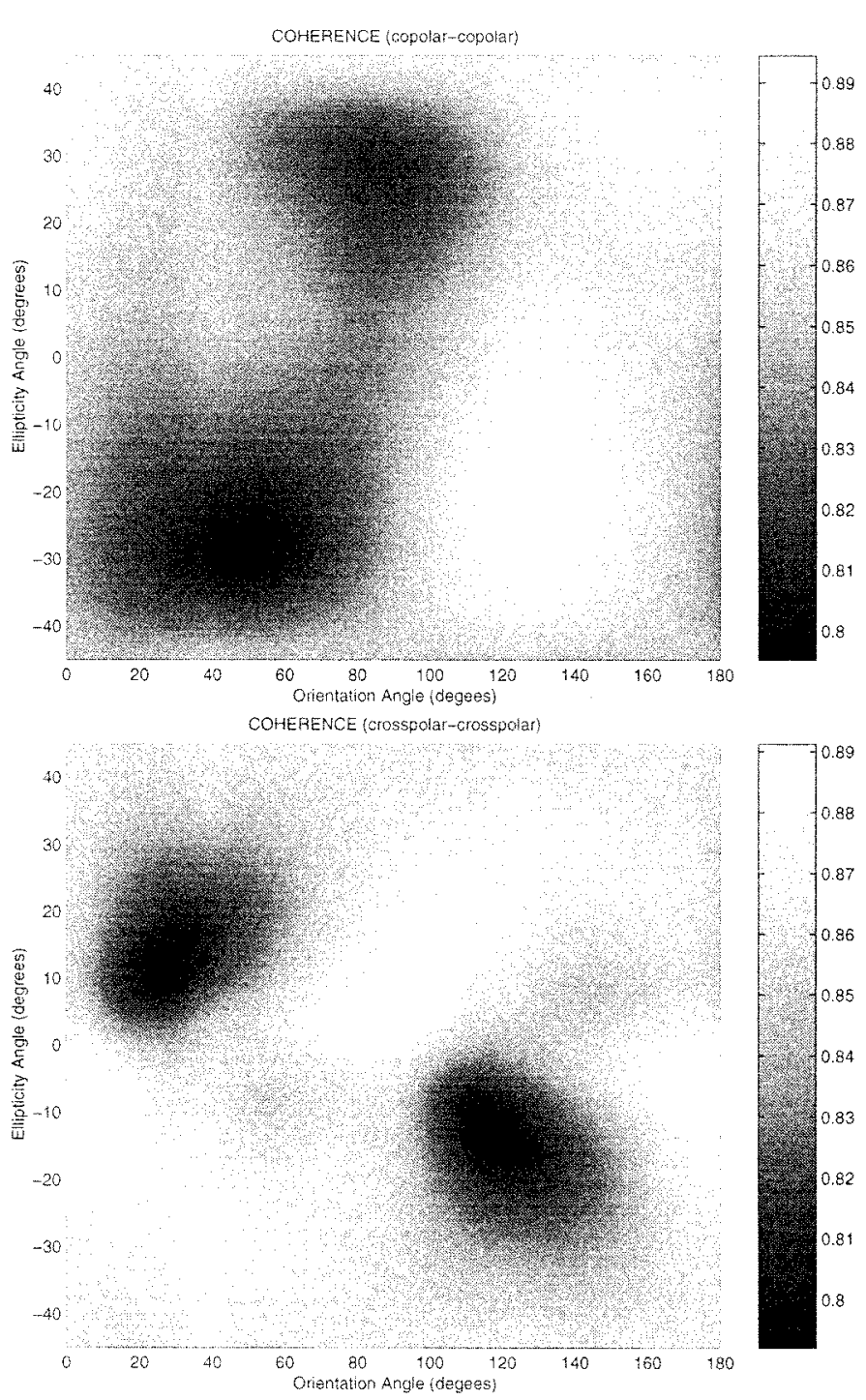

Fig. 9. Coherence maps for all $(\tau, \phi)$ polarization states, corresponding to the maize sample at $3.5 \mathrm{GHz}$, using a baseline $B=0.25^{\circ}$. Top: copolar $\gamma_{x x-x x}(\tau, \phi)$. Bottom: crosspolar $\gamma_{x y-x y}(\tau, \phi)$.

teria, six samples were taken in azimuth for all frequencies. A frequency averaging was also performed in the ensemble processing and multilook by using the samples at nine nonoverlapped bands, thus yielding 54 independent samples.

The height estimates at S-band and C-band for incidence angles from $44^{\circ}$ to $46^{\circ}$ are shown in Fig. 11. The displayed results were computed by applying only the polarimetric optimization algorithms. The retrieved values are very low. This is reasonable, because the scattering properties of such a target are homogeneous in the whole volume and so do not present a clear distribution of different behaviors as a function of height. Moreover, as justified in [28], for such a target, the backscatter return from the ground-trunk interactions at this incidence is very low in contrast to the response of the green needles. Finally, as suggested in a recent publication [30], for volumes with a random distribution of particles (without any preferred orientation), polarimetry has a little contribution in this estimation method. This is also true because of the low return from the ground. Instead,
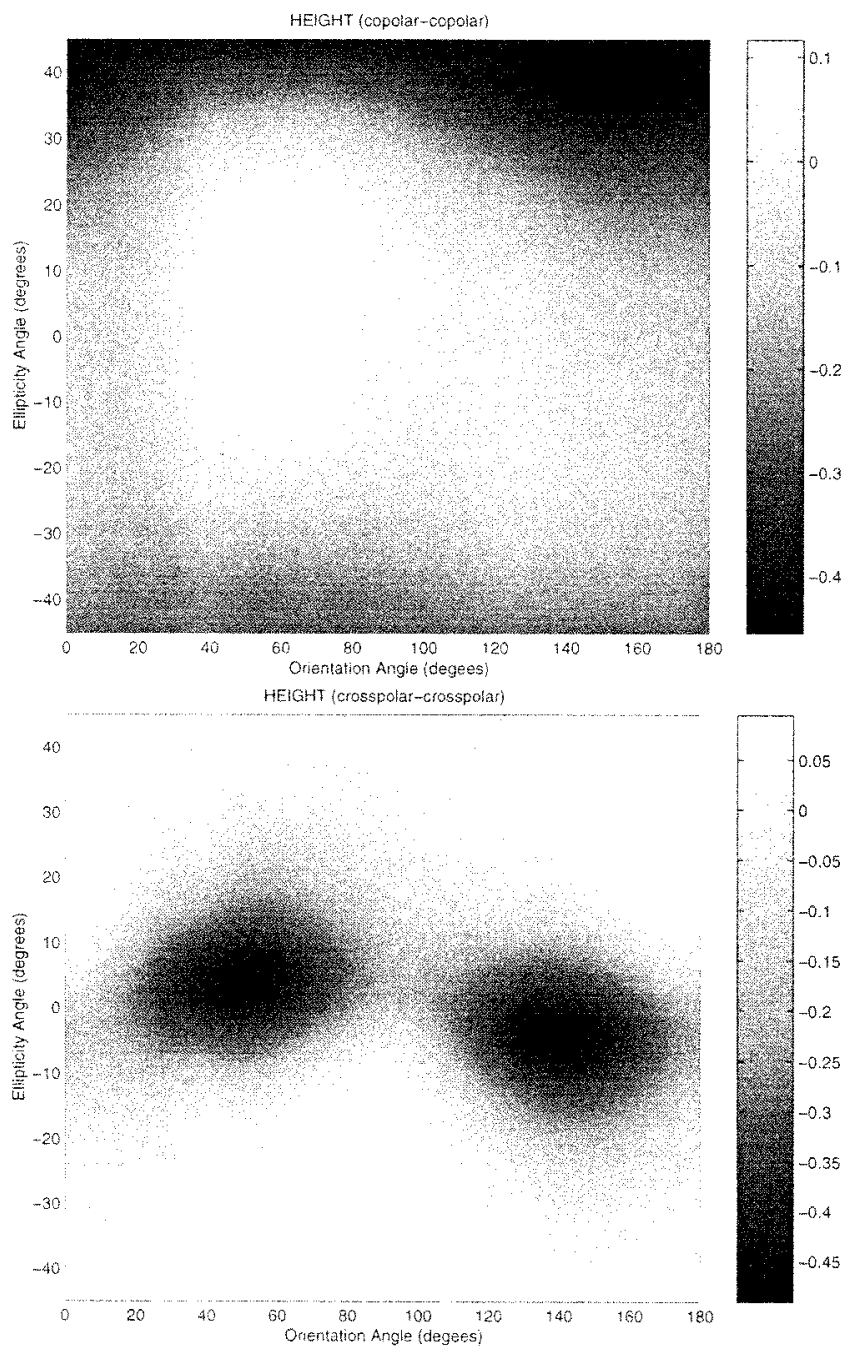

Fig. 10. Height maps for all $(\tau, \phi)$ polarization states, corresponding to the maize sample at $3.5 \mathrm{GHz}$, using a baseline $B=0.25^{\circ}$. Top: Copolar $\gamma_{x x-x x}(\tau, \phi)$. Bottom: Crosspolar $\gamma_{x y-x y}(\tau, \phi)$.

TABLE II

Height Estimates FoR the MAIZE SAMPLE (IN METERS)

\begin{tabular}{c|c|c|c|c}
\hline & \multicolumn{2}{|c|}{$B=0.25^{\circ}$} & \multicolumn{2}{c}{$B=0.5^{\circ}$} \\
\hline Frequency & PSM & Pol. Optimization & PSM & Pol. Optimization \\
\hline $1.5 \mathrm{GHz}$ & 0.18 & 0.54 & 0.19 & 0.50 \\
$3.5 \mathrm{GHz}$ & 0.40 & 0.20 & 0.43 & 0.30 \\
$5.5 \mathrm{GHz}$ & 0.41 & 0.18 & 0.44 & 0.67 \\
\hline
\end{tabular}

for a very oriented volume as maize is, this method is expected to work better. Note that a first estimation of a parameter related to the differential extinction coefficient between orthogonal polarizations (which is fundamental for the success of this technique [30]) was presented in [28].

The last experiment corresponds to the rice sample. This sample is shorter than the other two (only $60 \mathrm{~cm}$ high), so it is not expected to present ambiguities at high frequencies. As in the case of the cluster of fir trees, the measurement was originally planned for 3-D ISAR imaging and thus, the ensemble is performed by combining both frequency and spatial averaging. The frequency ranged from 1.5 to $9.5 \mathrm{GHz}$, and the baseline 


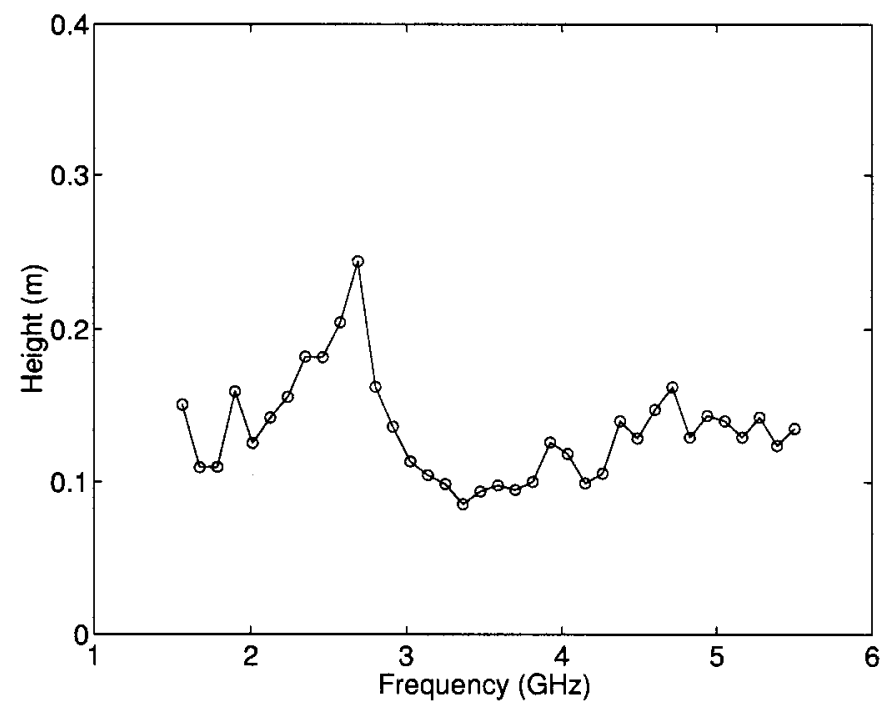

Fig. 11. Height estimates versus frequency for the cluster of young fir trees at $\mathrm{S}$-band and C-band. $B=0.5^{\circ}$.

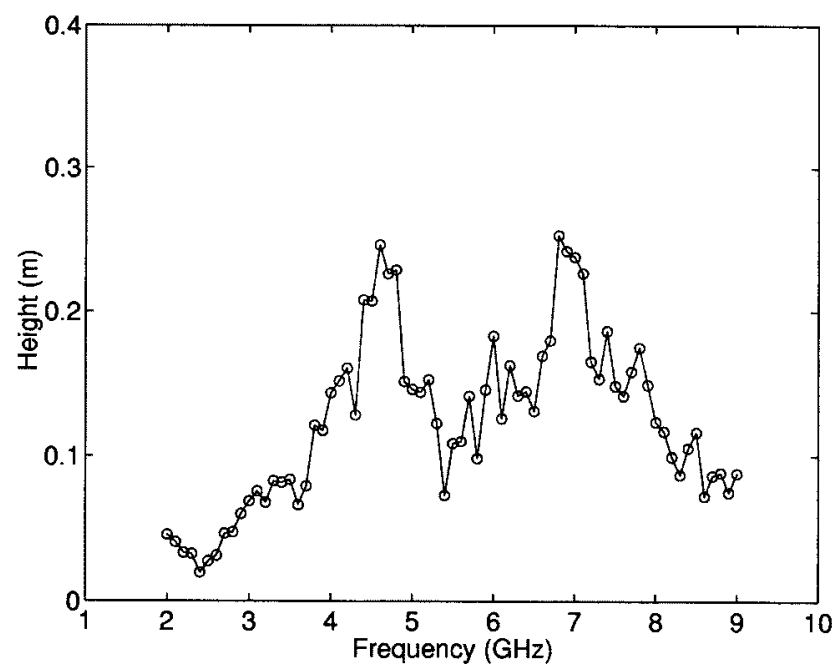

Fig. 12. Height estimates versus frequency for the sample of rice at S-band, C-band, and X-band. $B=0.5^{\circ}$.

was $0.5^{\circ}$. The bandwidth employed in the image reconstruction was $80 \mathrm{MHz}$. There were three independent samples by azimuth rotation at each frequency, and nine frequency sub-bands were used in the averaging, thus leading to 27 independent samples. As a result, the final estimates present a higher variance. Fig. 12 shows the estimates for S-band, C-band, and X band. The selected elevation incidence angles are $44^{\circ}$ to $46^{\circ}$, for comparison with the previous examples.

The estimates are within the expected range, but present strong relative variations between frequencies. For example, at $2-3 \mathrm{GHz}$, the retrieved height is almost zero, whereas at around 4.5 and $7 \mathrm{GHz}$, it is about $25 \mathrm{~cm}$. In general, it can be anticipated that at low frequencies, the estimates may be less reliable, because the backscatter return is strongly dominated by the ground-stem interaction, whereas the response from the above-ground leaves is quite low. At higher frequencies, the leaves start to exhibit a high response, so the target backscatter presents two scattering mechanisms (ground-stem and leaves)
TABLE III

Mean Vegetation Height Estimations (IN METERS)

\begin{tabular}{c|cc|c|c}
\hline Sample & \multicolumn{2}{|c|}{ Maize } & Fir trees & Rice \\
\hline Baseline & $0.25^{\circ}$ & $0.5^{\circ}$ & $0.5^{\circ}$ & $0.5^{\circ}$ \\
\hline L band & 0.47 & 0.43 & N.A. & N.A. \\
S band & 0.32 & 0.33 & 0.14 & 0.07 \\
C band & 0.21 & 0.57 & 0.13 & 0.15 \\
X band & N.A. & N.A. & N.A. & 0.14 \\
\hline
\end{tabular}

at different heights, and the retrieval procedure may yield better results.

All the results on vegetation height retrieval obtained by the coherence optimization are summarized in Table III. Only the average values are presented, because the variance is dependent on the type of ensemble averaging, which was different for each experiment. From the interpretation of the results in this section, it is evident that some a priori knowledge of the target morphology is needed to convert the estimated heights into absolute heights of the samples. This is because the method only measures the height difference between the scattering centers of the layers into which the target is decomposed, not the height difference between the top of the plants and the ground.

\section{CONCLUSION}

A recently published algorithm for optimizing the interferometric coherence by means of polarimetric information has been applied to laboratory experiments about two different problems: generation of DEM of nonvegetated surfaces and estimation of vegetation height. The results show that the height error in DEM generation can be drastically reduced within a wide range of baselines by using the optimization algorithm. With respect to vegetation, the presented results have been validated against ground truth data. The algorithm can estimate height without a DEM, since it measures the relative height differences between layers of plants or trees. In addition, an alternative simplified approach of the optimization algorithm has been presented for illustrating the working principle of polarimetry with application to interferometry.

Regarding the extraction of vegetation height, aspects such as the effect of multiple scattering on the retrieved position of the phase centers and the influence of differential extinction coefficient between orthogonal polarizations have not yet been analyzed. In order to study these topics, numerical and/or analytical models for different kinds of vegetation must be employed. Moreover, a deeper analysis of the precise relationship between the architecture of the samples (dielectric geometry) and the retrieved heights is needed for a correct interpretation and justification of the results. In order to do that, an alternative selection of scattering mechanisms to form the interferograms, based on the physical properties of the target and not on the coherence optimization, could be employed. For example, the elements of the scattering matrix ( $\mathrm{HH}, \mathrm{HV}$, and VV), or the Pauli spin matrices $(\mathrm{HH}+\mathrm{VV}, \mathrm{HH}-\mathrm{VV}, \mathrm{HV})$, may be chosen because they exhibit a clear physical meaning (odd bounces, even bounces, etc.).

Further outdoor work is planned to evaluate the coherence optimization technique when temporal decorrelation is present. 
This measurement campaign will use the fully polarimetric wide-band scatterometer developed at the Universitat Politècnica de Catalunya (UPC), Barcelona, Spain [31].

Additional measurements are also being planned at the European Microwave Signature Laboratory, Ispra, Italy, in order to study the application of the proposed coherence optimization for subsurface imaging applications (like mine detection) and for other issues related to SAR interferometry (multibaseline configurations, differential interferometry, phase unwrapping enhacement, etc.).

\section{ACKNOWLEDGMENT}

The authors would like to thank the personnel of the European Microwave Signature Laboratory, Ispra, Italy, for their support in performing the measurements and providing the experimental data. This work was carried out within the framework of a Training and Mobility of Researchers Network of the European Commision (TMR Network FMRX-CT98-0211).

\section{REFERENCES}

[1] R. Bamler and P. Hartl, "Synthetic aperture radar interferometry," Inv. Prob., vol. 14, pp. R1-R54, 1998.

[2] A. K. Gabriel, R. M. Goldstein, and H. A. Zebker, "Mapping small elevation changes over large areas: Differential radar interferometry," $J$. Geophys. Res., vol. 94, no. B7, pp. 9183-9191, 1989.

[3] H. A. Zebker, P. A. Rosen, R. M. Goldstein, A. K. Gabriel, and C. L. Werner, "On the derivation of coseismic displacement fields using differential radar interferometry: The Landers earthquake," J. Geophys. Res., vol. 99, no. B10, pp. 19617-19634, 1994.

[4] F. Gatelli, A. M. Guarnieri, F. Parizzi, P. Pasquali, C. Prati, and F. Rocca, "The wavenumber shift in SAR interferometry," IEEE Trans. Geosci. Remote Sensing, vol. 32, pp. 855-864, July 1994.

[5] M. Cattabeni, A. M. Guarnieri, and F. Rocca, "Estimation and improvement of coherence in SAR interferograms," in Proc. IEEE Int. Geosci. Remote Sensing Symp. (IGARSS), vol. 2, Pasadena, CA, Aug. 1994, pp. $720-722$.

[6] S. R. Cloude and E. Pottier, "An entropy based classification scheme for land applications of polarimetric SAR," IEEE Trans. Geosci. Remote Sensing, vol. 35, pp. 68-78, Jan. 1997.

[7] A. Freeman and S. L. Durden, "A three-component scattering model for polarimetric SAR data," IEEE Trans. Geosci. Remote Sensing, vol. 36, pp. 963-973, May 1998.

[8] D. L. Schuler, J.-S. Lee, and G. De Grandi, "Measurement of topography using polarimetric SAR images," IEEE Trans. Geosci. Remote Sensing, vol. 34, pp. 1266-1276, Sept. 1996.

[9] S. R. Cloude and K. P. Papathanassiou, "Polarimetric SAR interferometry," IEEE Trans. Geosci. Remote Sensing, vol. 36, pp. 1551-1565, Sept. 1998.

[10] T. Le Toan and N. Floury, "On the retrieval of forest biomass from SAR data," in Proc. 2nd Int. Symp. Retrieval of Bio- and Geo-physical Parameters from SAR Data for Land Applications, ESTEC, Noordwijk, The Netherlands, Oct. 1998, pp. 595-600.

[11] J. O. Hagberg, L. M. H. Ulander, and J. I. H. Askne, "Repeat-pass SAR interferometry over forested terrain," IEEE Trans. Geosci. Remote Sensing, vol. 33, pp. 331-340, Mar. 1995

[12] J. I. H. Askne, P. B. G. Dammert, L. M. H. Ulander, and G. Smith, "C-band repeat-pass interferometric SAR observations of the forest," IEEE Trans. Geosci. Remote Sensing, vol. 35, pp. 25-35, Jan. 1997.

[13] R. N. Treuhaft, S. N. Madsen, M. Moghaddam, and J. J. van Zyl, "Vegetation characteristics and underlying topography from interferometric radar," Radio Sci., vol. 31, pp. 1449-1485, Nov. 1996.

[14] K. P. Papathanassiou and S. R. Cloude, "Phase decomposition in polarimetric SAR interferometry," in Proc. IEEE Int. Geoscience and Remote Sensing Symp. (IGARSS), vol. 4, Seattle, WA, July 1998, pp. 2184-2186.

[15] K. P. Papathanassiou, A. Reigber, R. Sheiber, R. Horn, A. Moreira, an S. R. Cloude, "Airborne polarimetric SAR interferometry," in Proc. IEEE Int. Geoscience and Remote Sensing Symp. (IGARSS), vol. 4, Seattle, WA, July 1998, pp. 1901-1903.
[16] J. Gabriel, C. Schmullius, K. P. Papathanassiou, D. Darizhapov, G. Tathkov, T. Tsybjitov, and W. -M. Boerner, "Tree height extraction using polarimetric SAR interferometry," in Proc. IEEE Int. Geoscience and Remote Sensing Symp. (IGARSS), vol. 4, Hamburg, Germany, June 1999, pp. 2131-2133.

[17] W. Wiesbeck and D. Kähny, "Single reference, three target calibration and error correction for monostatic, polarimetric free space measurements," Proc. IEEE, vol. 79, pp. 1551-1558, Oct. 1991.

[18] A. J. Sieber, "The European microwave signature laboratory," EARSel Advances in Remote Sensing, vol. 2, pp. 195-204, Jan. 1993.

[19] L. Sagués, M. Bara, X. Fàbregas, and A. Broquetas, "A study of spectral decorrelation sources in wide band interferometry," in Proc. PIERSWorkshop on Advances in Radar Methods, Baveno, Italy, July 1998, pp. 64-66.

[20] H. A. Zebker and J. Villasenor, "Decorrelation in interferometric radar echoes," IEEE Trans. Geosci. Remote Sensing, vol. 30, pp. 950-959, Sept. 1992.

[21] J. Fortuny, E. Ohlmer, A. J. Sieber, P. Pasquali, C Prati, and F. Rocca, "Validating SAR interferometry applications by using EMSL," in Proc. IEEE Int. Geoscience and Remote Sensing Symp. (IGARSS), vol. 2, Pasadena, CA, Aug. 1994, pp. 736-738.

[22] L. Sagués, M. Bara, X. Fàbregas, and A. Broquetas, "Degradation of SAR interferometric coherence in wide band systems," J. Electromag. Waves Appl., vol. 14, pp. 185-204, Feb. 2000.

[23] S. R. Cloude and E. Pottier, "A review of target decomposition theorems in radar polarimetry," IEEE Trans. Geosci. Remote Sensing, vol. 34, pp. 498-518, Mar. 1996.

[24] X. Fàbregas, "Localización y Clasificación Polarimétrica de Centros de Dispersión en Blancos Complejos mediante Imágenes Radar.," Ph.D. thesis, Univ. Politècnica de Catalunya (UPC), Barcelona, Spain, 1995.

[25] J. Fortuny and A. J. Sieber, "Fast algorithm for a near-field synthetic aperture radar processor," IEEE Trans. Antennas Propagat., vol. 42, pp. 1458-1460, Oct. 1994.

[26] A. A. Monakov, J. Vivekanandan, A. S. Stjernman, and A. K. Nyström, "Spatial and frequency averaging techniques for a polarimetric scatterometer system," IEEE Trans. Geosci. Remote Sensing, vol. 32, pp. 187-196, Jan. 1994.

[27] K. Sarabandi, " $\Delta k$-radar equivalent to interferometric SAR's: A theoretical study for determination of vegetation height," IEEE Trans. Geosci. Remote Sensing, vol. 35, pp. 1267-1276, Sept. 1997.

[28] J. M. Lopez-Sanchez, J. Fortuny, S. R. Cloude, and A. J. Sieber, "Indoor polarimetric radar measurements on vegetation samples at $\mathrm{L}, \mathrm{S}, \mathrm{C}$ and X band,” J. Electromagn. Waves Appl., vol. 14, pp. 205-231, Feb. 2000.

[29] K. Sarabandi, C. G. Brown, and L. Pierce, "Tree height estimation from the polarimetric and interferometric radar response," in Proc. IEEE Int. Geoscience and Remote Sensing Symp. (IGARSS), vol. 4, Hamburg, Germany, June 1999, pp. 1986-1988.

[30] R. N. Treuhaft and S. R. Cloude, "The structure of oriented vegetation from polarimetric interferometry," IEEE Trans. Geosci. Remote Sensing, vol. 37, pp. 2620-2624, Sept. 1999.

[31] L. Sagués, M. Bara, O. Mora, A. Broquetas, and X. Fàbregas, "Circular synthetic aperture radar (C-SAR) system and an interferometric algorithm for ground-based applications," in Proc. EUSAR, Friedrichshafen, Germany, May 1998, pp. 499-502.

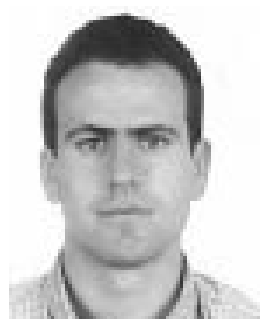

Lluís Sagués (S'98) was born in Barcelona, Spain, in 1972. He received the Ingeniero degree in telecommunication engineering with a thesis on ground-based SAR systems, from the Polytechnic University of Catalonia (UPC), Barcelona, Spain in 1996, where he is currently pursuing the Ph.D. degree.

Since 1997, he has been with the Electromagnetics and Photonics Engineering Group, Department of Signal Theory and Communications, UPC, working on radar polarimetry and SAR interferometry. His reasearch interests include radar imaging, physical parameters retrieval of vegetated and nonvegetated terrains, and buried object detection. 


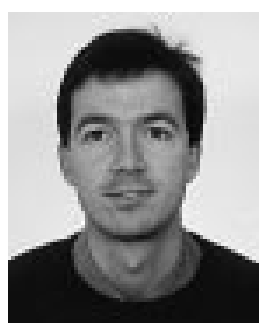

Juan M. Lopez-Sanchez (S'98) was born in Alicante, Spain, in 1972. He received the Ingeniero degree in telecommunications engineering from the Polytechnic University of Valencia (UPV), Valencia, Spain, in 1996, where he is currently pursuing the $\mathrm{Ph} . \mathrm{D}$. degree under a predoctoral grant from the Joint Research Centre, European Commission, Ispra, Italy.

From 1994 to 1997, he worked at the Communications Department, UPV, in numerical methods for electromagnetics, focusing on spectral techniques applied to antenna design and analysis, and to propagation models. His main research interests include analytical and numerical models for multiple scattering problems, microwave remote sensing for inversion of biophysical parameters, polarimetric techniques, and SAR imaging algorithms.

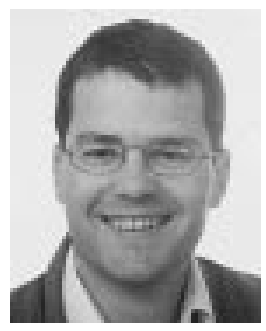

Joaquim Fortuny (S'93-A'96) was born in Tarragona, Spain, in 1964. He received the Ingeniero degree in telecommunications engineering from the Polytechnic University of Catalonia (UPC), Barcelona, Spain, in 1988.

From 1988 to 1989 , he worked on the design of microwave circuits at Ka-Band in the Antennas, Microwave, and Radar group, UPC. From 1990 to 1992, he worked as a Research Assistant in the RF Division, European Space Technology Centre, European Space Agency, The Netherlands. Since 1993, he has been with the Joint Research Centre, European Commission, Ispra, Italy. His research interests are in radar imaging, subsurface sensing, and numerical techniques in electromagnetics.

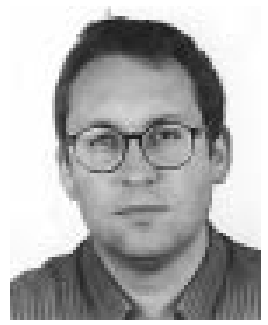

Xavier Fàbregas (M'98) received the B.S. degree in physics from the Barcelona University, Barcelona, Spain, in 1988, and the Ph.D. degree in applied sciences from the Polytechnic University of Catalonia (UPC), Barcelona, Spain, in 1995.

Since 1996, he has been Associate Professor with UPC. His current research interests include polarimetric retrieval algorithms, polarimetric calibration, and polarimetric SAR classification.

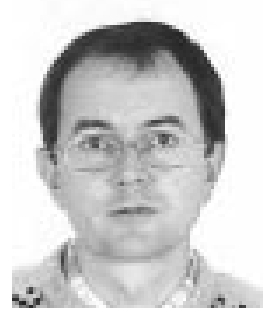

Antoni Broquetas (S'98-M'89) was born in Barcelona, Spain, in 1959. He received the Ingeniero degree in telecommunication engineering from the Polytechnic University of Catalonia (UPC), Barcelona, Spain, in 1985, and the Doctor Ingeniero degree in 1989 in telecommunications engineering for his work on microwave tomography, from the UPC as well.

In 1986, he was a Research Assistant in the Portsmouth Polytechnic, U.K., involved in propagation studies. In 1987, he joined the Department of Signal Theory and Communications, School of Telecommunication Engineering, UPC. Currently, he is an Associate Professor with the UPC, involved in research on radar imaging and remote sensing and responsible of the research activities of the recently created Institute of Geomatics.

Alois J. Sieber (M'80-SM'86) received the M.S. degree in physics from the Technical University of Karslruhe, Karlsruhe, Germany, in 1971, the Ph.D. degree in 1973 from the University of Tübingen, Tübingen, Germany, and the Habilitation degree in remote sensing in 1985 from the University of Stuttgart, Stuttgart, Germany.

Since 1986, he has been working at the Joint Research Centre, European Commission, Ispra, Italy. He is the Head of the Technologies for Detection and Positioning Unit, Space Applications Institute. Among other activities, he is also the head of the European Microwave Signature Laboratory (EMSL), Ispra, Italy. Since 1986, he has been a Senior Lecturer, University of Stuttgart.

His research interests are in remote sensing, radar imaging, subsurface sensing, and numerical techniques in electromagnetics. He is leading several projects supporting the goal to overcome the global scourge of landmines within the foreseeable future. 